\title{
Advanced Visualization and Interaction Systems for Surgical Pre-operative Planning
}

\author{
Lucio T. De Paolis ${ }^{1}$, Marco Pulimeno ${ }^{2}$ and Giovanni Aloisio ${ }^{1}$ \\ ${ }^{1}$ Department of Innovation Engineering, University of Salento, Lecce, Italy \\ ${ }^{2}$ Faculty of Engineering, University of Salento, Lecce, Italy
}

The visualization of 3D models of the patient's body emerges as a priority in surgery. In this paper two different visualization and interaction systems are presented: a virtual interface and a low cost multi-touch screen. The systems are able to interpret in real-time the user's movements and can be used in the surgical pre-operative planning for the navigation and manipulation of 3D models of the human body built from CT images. The surgeon can visualize both the standard patient information, such as the CT image dataset, and the 3D model of the patient's organs built from these images. The developed virtual interface is the first prototype of a system designed to avoid any contact with the computer so that the surgeon is able to visualize models of the patient's organs and to interact with these, moving the finger in the free space. The multi-touch screen provides a custom user interface developed for doctors' needs that allows users to interact, for surgical pre-operative planning purposes, both with the 3D model of the patient's body built from medical images, and with the image dataset.

Keywords: user interface, medical imaging, multi-touch screen, surgical planning

\section{Introduction}

Modern medical imaging provides an accurate knowledge of patient's anatomy and pathologies and, even though the information interpretation of the computed tomography (CT) and the magnetic resonance images (MRI) remains a difficult task, image processing methods, highspeed graphic workstations and virtual reality techniques have expanded the possibilities in the area of diagnosis and treatment, making it possible to localize the pathologies more accurately and to see the anatomic relationships like never before.
Minimally Invasive Surgical (MIS) procedures could greatly benefit from the visualization of 3D models of the specific patient's organs and the introduction of new interaction modalities with such models could allow the surgeon to get all visual information he needs for a more accurate diagnosis and for a more detailed surgical pre-operative planning.

Several research teams have been dealt with in the development of advanced modalities of interaction and visualization in many application fields, and various gesture-based interfaces, some of these in medical applications, have been developed; the tracked movements of the fingers provide a more natural and less-restrictive way of 3D model manipulating.

Grätzel et al. [3] have presented a non-contact mouse for surgeon-computer interaction in order to replace the standard computer mouse functions with the hand gestures.

Wachs et al. [17] have presented Gestix, a visionbased hand gesture capture and recognition system for the navigation and manipulation of images.

O'Hagan and Zelinsky [10] have presented a prototype of interface, based on a tracking system, where a finger is used as a pointing and a selection device.

The collaboration between the MIT Artificial Intelligence Lab and the Surgical Planning Laboratory of Brigham [4] has led to the development of solutions that support the pre-operative 
surgical planning and the intra-operative surgical guidance.

Hartmut et al. [6] have described the integration of image analysis methods with a commercial image-guided navigation system for neurosurgery (the BrainLAB Vector Vision Cranial System).

Feied et al. [2] have developed a hand-free system to review digital radiologic images during a clinical procedure so that the clinicians can avoid the contact with the keyboards and mice that are potential sources for contamination.

Vilimek and Zander [16] have combined eyegaze input with a brain-computer interface in order to obtain a more reliable and less error prone contactless interaction. The multimodal interface involves eye movements to determine the object of interest and a Brain-Computer Interface to simulate the mouse click.

Cheng et al. describe [1] the use of textile, multielectrode capacitive on body sensing for contactless detection of simple control gestures in a hospital ward scenario. The focus is the design, implementation, and evaluation of a sensing system and the detection of gestures with the multi-electrode design.

Ishikawa et al. [7] have introduced a touchless input device and gesture commands for operating a PC that negates the need to touch it or wear input devices to use it. They have used distance sensor to capture gestures and this solution makes the device very simple. The system is practical enough to use for viewer operation, so it is applicable for not only PCs, but also audiovisual devices like TVs and HDD recorders.

W. Gu at al. [5] have developed a touchless infrared-tracking interface for image viewing and manipulation. The system can be tuned on the needs of the doctors and is based on an infrared camera and an image-processing unit in combination with modular pointing and clicking devices. The interchangeable pointing devices include retro-reflectors that can be incorporated onto gloves, surgical tools or head wear and allow for hands-free mouse cursor control if desired. Clicking can be performed via foot control, manual clicker or voice control.

In this paper, we present the first prototypes of two advanced visualization and interaction systems used for the surgical pre-operative plan- ning: a virtual interface and a low cost multitouch screen. The systems are able to visualize the 3D model of a patient's body built from a medical images dataset, to interpret in real-time the user's movements and to provide the possibility of interaction with the 3D models of the organs.

This work is part of the ARPED Project (Augmented Reality Application in Pediatric Minimally Invasive Surgery) that has been funded by the Fondazione Cassa di Risparmio di Puglia. The aim of the ARPED Project is the design and development of an Augmented Reality system that can support a surgeon involved in a laparoscopic surgical procedure.

\section{The 3D Models of the Organs}

An efficient 3D reconstruction of the patient's anatomy can be provided from his medical image (MRI or CT) in order to improve the standard slice view by the visualization of 3D models of the organs. Some segmentation and classification algorithms have been applied in order to distinguish the different anatomical structures; the grey levels in the medical images are replaced with colors associated to the organs.

Currently, there are different image processing tools used for the visualization of medical images and for the 3D modelling of human organs; some of these tools are commercial and other are open source. Some of the most important are Mimics [9], 3D Slicer [19], OsiriX [12] and ITK-SNAP [18].

In the developed virtual interface we have utilized 3D Slicer for building the 3D models of the organs from a CT dataset of images. 3D Slicer is a multi-platform open-source software package for the visualization and image analysis; the platform provides functionality for the segmentation and three-dimensional visualization of multi-modal image data, as well as advanced image analysis algorithms for the diffusion tensor imaging, the functional magnetic resonance imaging and the image-guided therapy. Standard image file formats are supported.

In our application, the 3D models of the abdominal area have been reconstructed and, in order to obtain information about the size and 
the shape of the human organs, some segmentation and classification algorithms have been used. In particular, the Fast Marching algorithm has been used for the image segmentation and some fiducial points have been chosen in the interest area and used in the growing phase [14].

After the first semi-automatic segmentation, a manual segmentation has been carried out.

A 3D model of the abdominal area, reconstructed from CT images, is shown in Figure 1. The patient suffers from a liver disease and this organ appears considerably swollen.

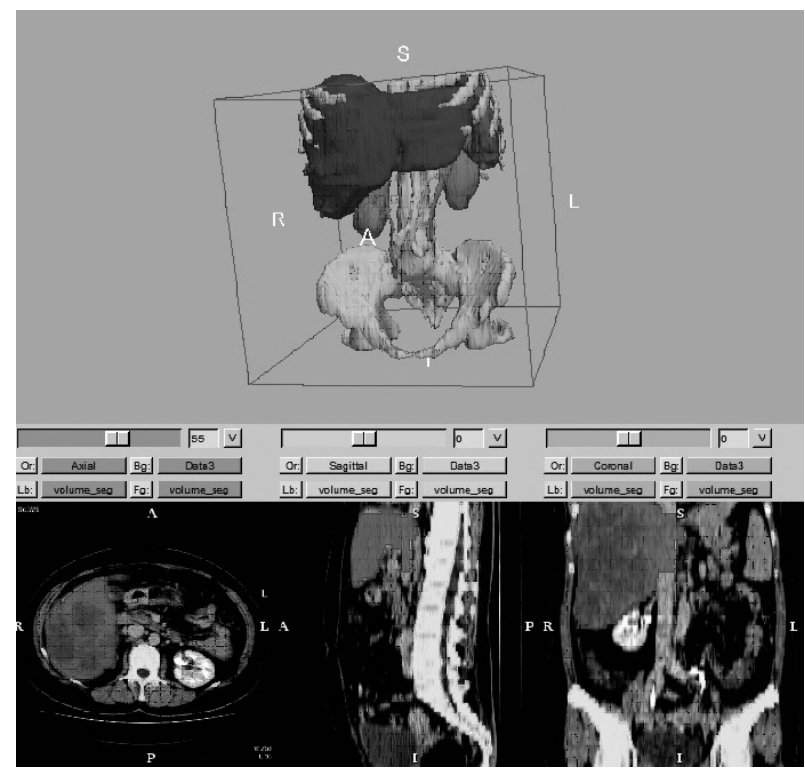

Figure 1. The reconstruction of the 3D model.

\section{The Virtual Interface}

\subsection{The used technologies}

In order to detect the user's finger position and to track his movements, an optical tracking system has been used, in particular, the Polaris Vicra of the NDI [13] has been chosen.

The device is able to track both active and passive markers and a position sensor is used to detect markers affixed to a tool or object; based on the information received, this sensor is able to determine position and orientation of the tools within a specific measurement volume. In this way, each movement of a marker (or marker geometry) in the real environment is replicated in the corresponding virtual environment.

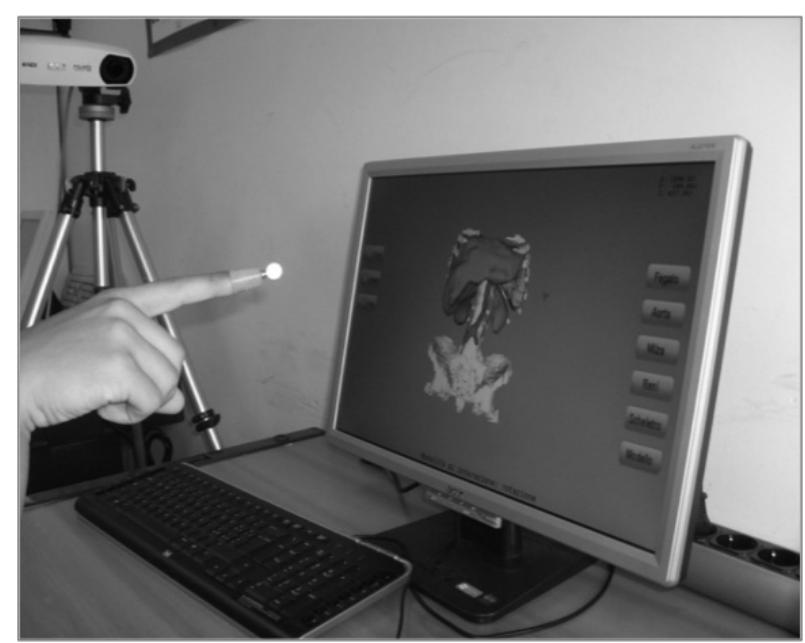

Figure 2. Use modality of the virtual interface.

Figure 2 shows the use modality of virtual interface and a single reflective sphere is used to track the finger movements by means of the optical tracker and to interact with the virtual interface.

OpenSceneGraph [11] that is an open source high performance 3D graphics toolkit available on multiple platforms has been utilized for the building of the graphic environment.

To build the virtual scene, a scenegraph has been used and the 2D and 3D environments have been included. Figure 3 shows the complete graph of the developed graphic scene.

The 2D environment allows visualizing the cursor, some text and the buttons; in addition, the active interaction modality and the cursor position are updated.

The 3D environment allows visualizing the model of the organs and providing the interaction operations (rotation, translation and zoom).

\subsection{The developed application}

The developed system is the first prototype of a virtual interface designed to avoid any contact with the computer so that the surgeon can visualize the models of the patient's organs and interact with these in a more effective way. 


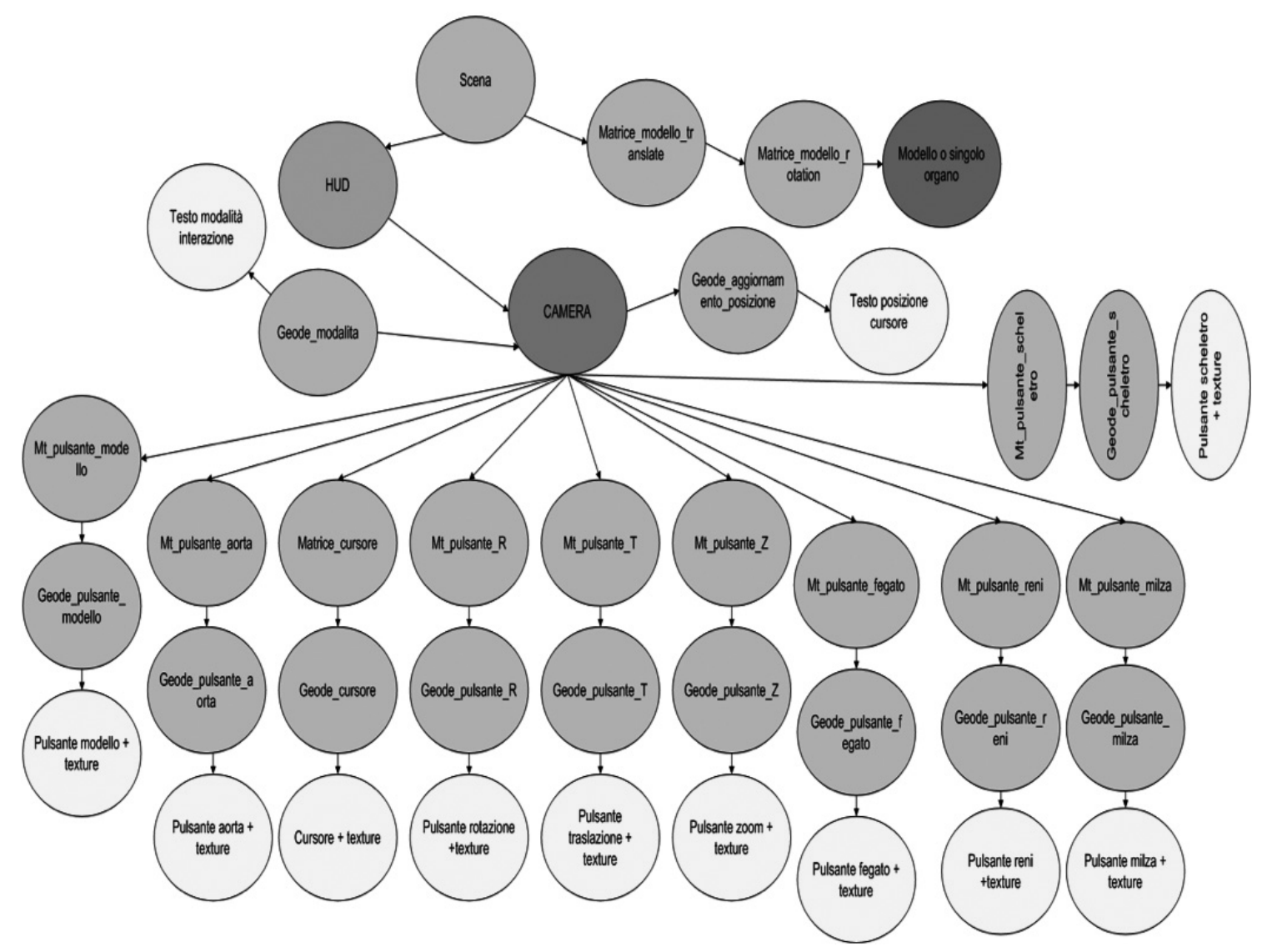

Figure 3. The graph of the graphic scene.

The system can be used for diagnosis, for surgical pre-operative planning and also during the real surgical procedure.

In modern operating rooms, the optical tracker already exists and, for this reason, the developed application can be used without any modification of the surgical environment.

Using the virtual interface, the interactions with the 3D models of the patient's organs happen in real-time and the interface appears as a touchscreen suspended in the free space; the position of the virtual interface is chosen by the user when the application is started up.

The choice of the space area where the interface has to be located is decided just specifying the positions of the four vertexes of the virtual screen. In this way the interaction plane is fixed and a reference system is also defined.

The finger movements are detected by means of the optical tracking system and are used to simulate the touch with the virtual interface.
In front of the area where the virtual interface has been placed, the user's finger is moved around; the interaction with the virtual interface happens just pressing the virtual buttons present on the interface on the left side; a textual information of the chosen modality is visualized in the screen on the bottom side.

Moving the finger in the free space, the results of the interaction are visualized in the central part of the screen.

Figure 4 shows the visualization on the screen of the developed user interface that allows visualization and interaction with the $3 \mathrm{D}$ models of the organs; the buttons for the choice of the interaction modality are located on the left side of the screen and the buttons for the selection of the organs on the right side.

In order to adapt the size of the virtual interface to the real screen of the computer, a scaling operation is carried out. 


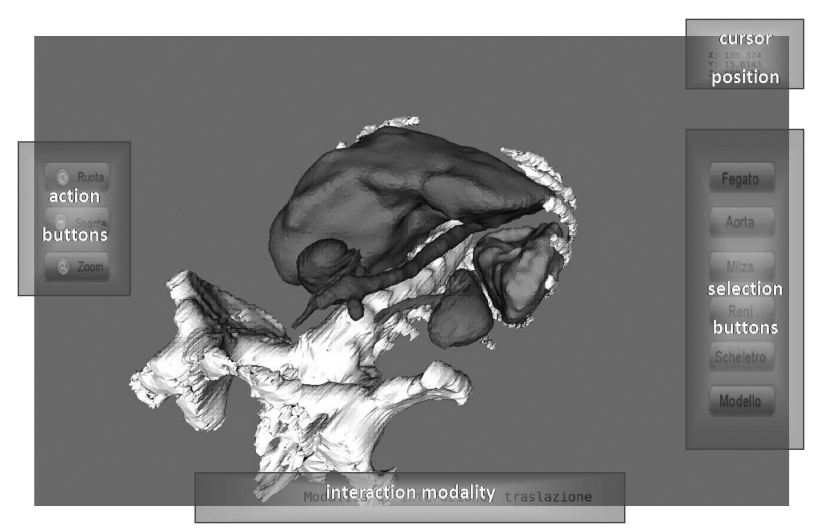

Figure 4. The user interface.

The user can choose different interaction modalities and decide which model has to be visualized. In particular, by pressing the user interface buttons on the right, it is possible to visualize the complete 3D model or a specific organ that is part of the 3D model; the buttons on the left make it possible to choose one of the interaction modalities. The allowed interaction modalities are translation, rotation and the zoom in or out.

At the bottom of the screen, the chosen interaction modality is visualized and in the top lefthand corner, the cursor position in the defined reference system is shown.

\section{The Multi-touch Screen}

The designed and developed multi-touch screen provides a user interface customized for doctors' requirements, allowing many users to interact at the same time, with 3D models of the human body built from CT images.

The system is based on the rear-side illumination technique that allows detection of the fingertips on the screen using some IR illuminators and an IR camera; this technique is shown in Figure 5.

In order to identify finger contacts with the screen and to translate these in specific events, the open-source and multi-platform TouchLib library [15], a C-based package from the NUI Group, has been used.

By means of a calibration phase and using specific filters, it was possible to eliminate any noise and optimize detection of the contact points coordinates of the fingers on the screen;

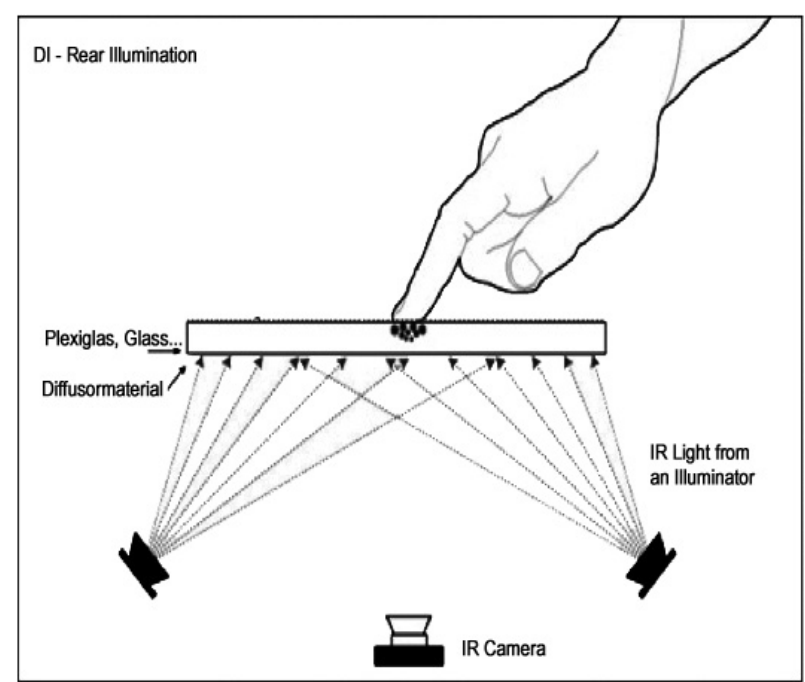

Figure 5. The rear-side illumination technique.

the user interface for the calibration procedure and filtering is shown in Figure 6.

Communication between the developed and the tracker applications happens by means of the TUIO [8], an open framework that defines a common protocol and API for tangible multitouch surfaces.

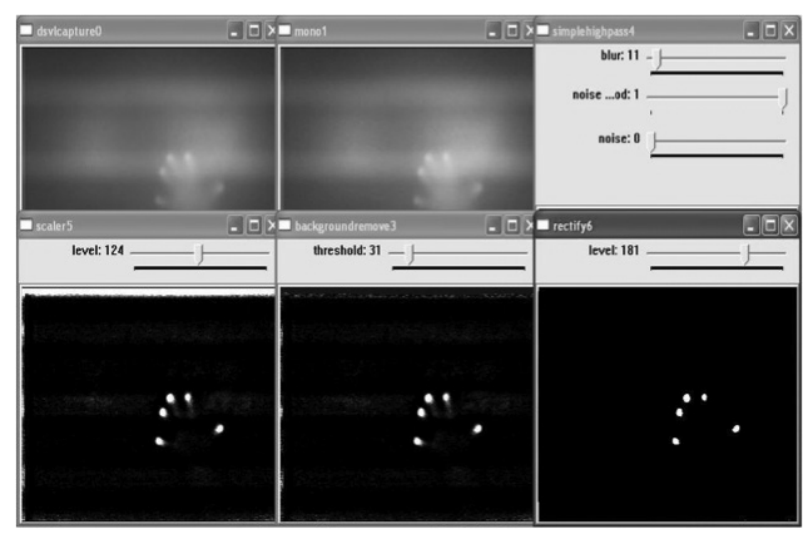

Figure 6. The calibration of the multi-touch screen.

The TUIO protocol provides a general and versatile communication interface between the tangible tabletop controller interfaces and the underlying application layers. TUIO is used by several libraries dedicated to tracking (such as Touchlib) and allows their inter-changeability. In this way the application appears to be a module independent from the tracker application in use. 


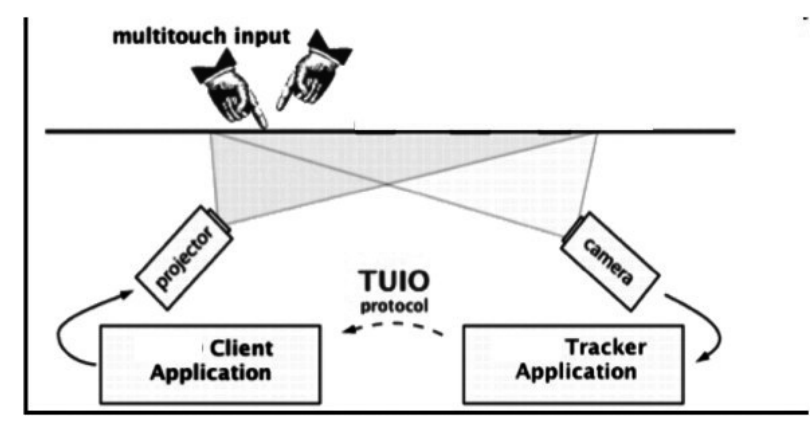

Figure 7. The touch screen working modality.

Figure 7 shows the working modality of the multi-touch screen where the user is able to do gestures onto the table surface with the fingertips; these gestures are associated to different interaction modalities with the $3 \mathrm{D}$ models.

The user interface of the multi-touch screen is provided with many buttons in order to visualize both the 3D models of the human organs and the CT slice sets used to build these virtual models.

The interaction with the models is possible using one finger (to rotate or translate) or two fingers (to zoom in or zoom out).

The use of the system results is very simple and evident for the user and the touch screen can be considered a helpful tool for the diagnosis and surgical pre-operation planning.

Figures 8 and 9 show some examples of the interaction with 3D models of the patient's organs, using one finger (in order to rotate or translate the model) and using two fingers (in order to zoom in the selected organ model).

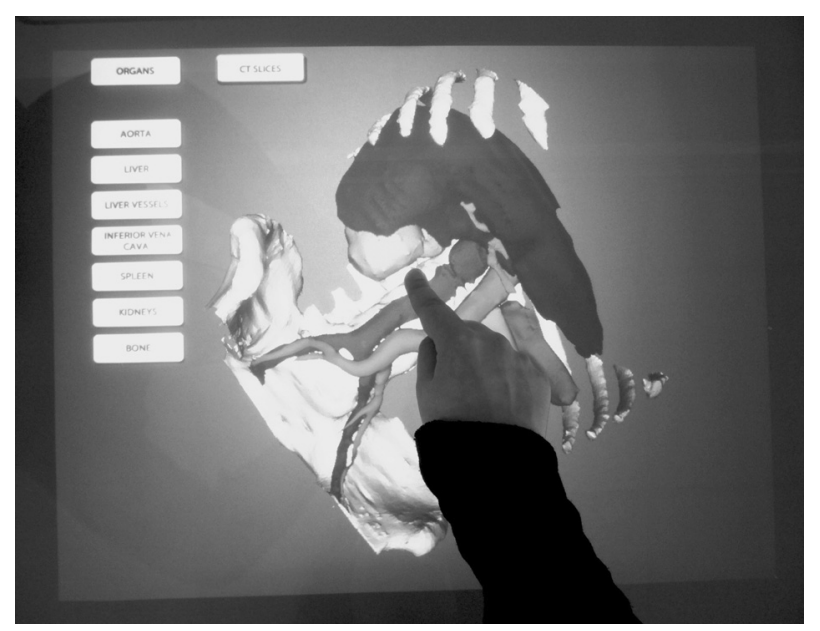

Figure 8. The interaction using one finger.

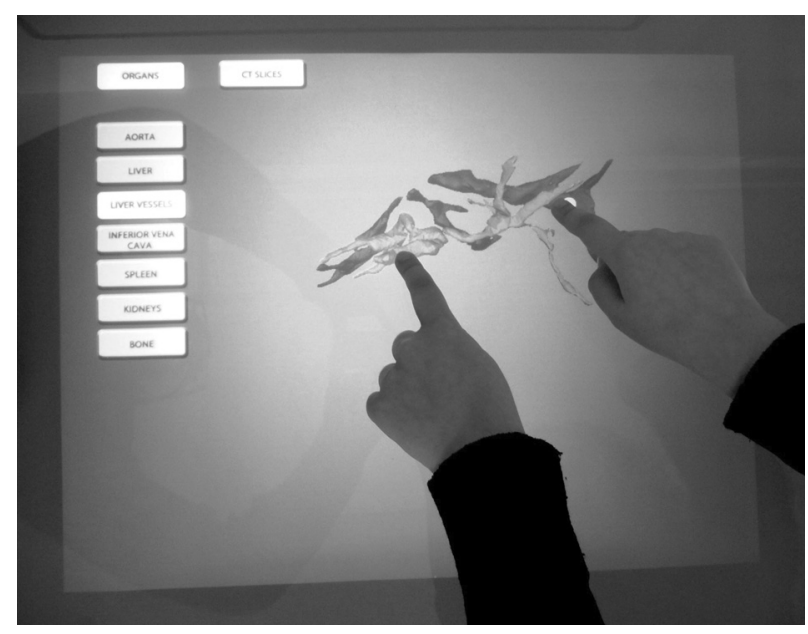

Figure 9. The interactions using two fingers.

In addition, it is possible to visualize a complete CT dataset of the patient's images used for building of the 3D model of their organs, choose the specific slice using the arrows and interact with this by using the same interaction modalities applied to the models. This situation is shown in Figure 10.

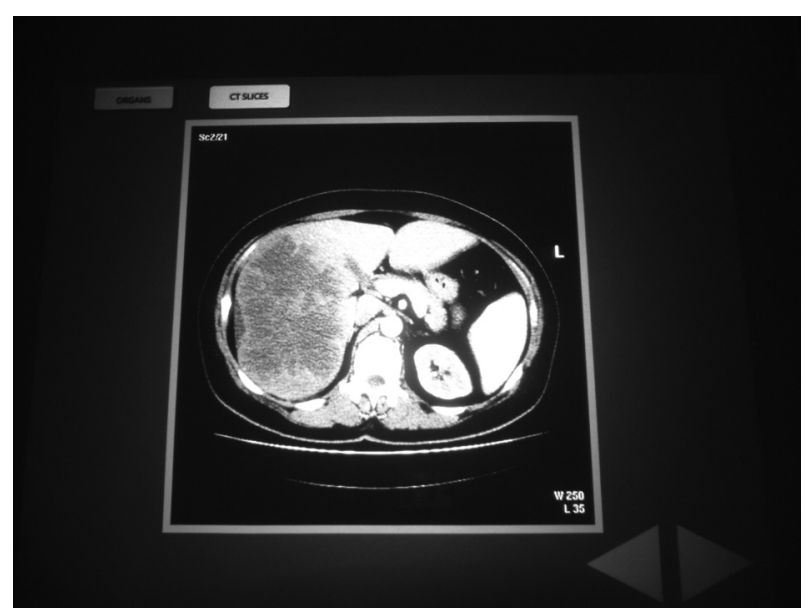

Figure 10. Visualization of the CT image set.

\section{Conclusions and Future Work}

In this paper we presented two advanced visualization and interaction systems used for the surgical pre-operative planning: a virtual interface and a low-cost multi-touch screen. 
The systems are able to interpret, in real time, the user's movements and to convert these into interactions with the 3D models of human organs built from the patient's CT images.

The developed virtual interface provides an interaction modality which is similar to the normal one used in the touch-screen systems, but there is no contact with the screen and the user's finger is moved in the free space.

The developed multi-touch screen provides a user interface customized for doctors' needs and the system is able to detect the position of up to two fingers; the system provides the surgeons with a means for the visualization and the interaction with both the standard patient information, such as the CT image dataset, and the 3D models of the patient's organs built from these images.

The introduction of other modalities of interaction with the 3D models is in progress, after further investigation and consideration of surgeons' requirements. Furthermore, verification of the interfaces usability and effectiveness in real deployment is planned.

In addition, taking into account possible use of the virtual interface with an optical tracker in the operating room during surgical procedures, the problem of possible undesired interferences due to the detection of false markers (phantom markers) will be evaluated.

\section{References}

[1] J. Cheng, D. Bannach, P. Lukowicz, On Body Capacitive Sensing for a Simple Touchless User Interface. Presented at the 5th International Workshop on Wearable and Implantable Body Sensor Networks, (2008), China, pp. 113-116.

[2] C. Feied, M. Gillam, J. Wachs, J. Handler, H. STERN, M. SMITH, A Real-time Gesture Interface for Hands-free Control of Electronic Medical Records. Presented at the AMIA Annual Symposium Proceedings, (2006).

[3] C. Grätzel, T. Fong, S. Grange, C. BAur, A Non-contact Mouse for Surgeon-Computer Interaction. Technology and Health Care Journal, IOS Press, (2004), 12(3).

[4] W. Grimson, G. EtTinger, T. Kapur, M. LeVenTON, W. WELLS, R. KIKINIS, Utilizing Segmented MRI Data in Image-guided Surgery. International Journal of Pattern Recognition and Artificial Intelligence, (1998), 11(8), pp. 1367-1397.
[5] W. Gu, D. Shen, K. ChaO, J. Wall, T. M. KrumMEL, F. FEINSTEIN, D. Y. SZE, R. GUZMAN, I. HOFMANN, Touchless Interface for Image Viewing and Manipulation during Interventional Procedures. Journal of Vascular and Interventional Radiology, 2(2009).

[6] K. Hartmut, D. C. Widenka, C. B. Lumenta, BrainLab Vector Vision Neuronavigation System: Technology and Clinical Experiences in 131 Cases. Neurosurgery, (1999), 44(1), pp. 97-104.

[7] T. IshiKawa, Y. Horry, T. Hoshino, Touchless Input Device and Gesture Commands. Presented at the International Conference on Digital Object Identifier, (2005), pp. 205-206.

[8] M. Kaltenbrumer, R. BEnCinA, T. BOVERMANN, E. CostanzA, Tuio: A protocol for table-top tangible user interfaces, Gesture in Human-Computer Interaction and Simulation. In Lecture Notes in Artificial Intelligence, (2006), Vol. 3881, SpringerVerlag.

[9] MIMICS Medical Imaging Software, Materialise. Information available online via http://www.materialise.com.

[10] R. O'Hagan, A. Zelinsky, Finger Track - A Robust and Real-time Gesture Interface. In Lecture Notes in Computer Science, (1997), vol.1342, Springer-Verlag.

[11] OPENSCENEGRAPH. Information available online via http: //www . openscenegraph.org.

[12] OSIRIX Imaging Software. Information available online via http://www.osirix-viewer.com.

[13] NDI POLARIS VICRA. Information available online via http: //www.ndigital.com.

[14] J. A. Sethian, A Fast Marching Level Set Method for Monotonically Advancing Fronts. PNAS (1996), 93(4), pp. 1591-1595.

[15] TOUCHLIB, A Multi-touch Development Kit. Information available online via http://www . nuigroup.com/touchlib.

[16] R. VilimeK, T. O. ZANDER, BC(eye): Combining Eye-gaze Input with Brain-Computer Interaction. In Lecture Notes in Computer Science, (2009), Vol. 5615 , pp. 593-602, Springer-Verlag.

[17] J. P. WaChS, H. I. Stern, Y. EdAN, M. Gillam, J. HANDLER, C. FEIED, M. A. SMITH, A Gesture-based Tool for Sterile Browsing of Radiology Images. The Journal of the American Medical Informatics Association, (2008), 15(3).

[18] P. A. Yushkevich, J. Piven, H. Cody, S. Ho, J. C. GEE, G. GERIG, User-guided Level Set Segmentation of Anatomical Structures with ITK-SNAP. Presented at the MICCAI Workshop on Open-source Software, (2005).

[19] 3D SLICER. Information available online via http://www.slicer.org. 
Received: June, 2010 Accepted: November, 2010

Contact addresses.

Lucio T. De Paolis

Department of Innovation Engineering

University of Salento

Lecce, Italy

e-mail: lucio.depaolis@unisalento.it

Marco Pulimeno

Faculty of Engineering

University of Salento

Lecce, Italy

e-mail: marco.pulimeno@unisalento.it

Giovanni Aloisio

Department of Innovation Engineering

University of Salento

Lecce, Italy

e-mail: giovanni.aloisio@unisalento.it
Lucio Tommaso DE PAOLIS is an assistant professor of information processing systems at the Department of Innovation Engineering of the University of Salento (Italy).

He received a degree in electronic engineering from the University of Pisa (Italy) and started his research at the Scuola Superiore S. Anna of Pisa and then continued at the University of Salento. His research interest concerns the study of the interactions in virtual environments and the development of human-computer interfaces. This study has been focused on the building of realistic simulators for surgical training and on the applications of Virtual Reality and Augmented Reality technologies in medicine and surgery.

De Paolis was a visiting researcher in 2007 and 2010 at the Centro de Ciencias Aplicadas y Desarrollo Tecnologico (CCADET) - Universidad Nacional Autonoma de Mexico (UNAM) - Mexico City (Messico) and in 2007 and 2009 at the Computer Graphics Laboratory - Sabanci University - Istanbul (Turkey). In 2010 De Paolis was a visiting professor at the University of Aalborg (Denmark).

$\mathrm{He}$ is a member of the SMIT (Society for Medical Innovation and Technology) and the MIMOS (Italian Movement of Modelling and Simulation).

He teaches computer science at the Sciences Faculty of the University of Salento.

GIOVANNI ALOISIO is full professor of information processing systems at the Engineering Faculty of the University of Salento, Lecce, Italy and head of the Division "Scientific Computing and Operations" (SCO) at the Euro-Mediterranean Center for Climate Change (CMCC).

His expertise concerns high performance computing, grid \& cloud computing and distributed data management. He was a co-founder of the European Grid Forum (Egrid) which then merged into the Global Grid Forum (GGF), now Open Grid Forum (OGF). He was involved in the EGEE EU FP5-FP6 grid projects (Enabling Grids for E-science, http://www.eu-egee.org/). He is responsible for CMCC of the EU-FP7 IS-ENES (InfraStructure for the European Network for Earth System modelling) project.

He is responsible for ENES of the EU-FP7 EESI (European Exascale Software Initiative) Project and member of the ENES HPC Task Force. $\mathrm{He}$ is a key expert of IESP (International Exascale Software Project), whose main goal is definition of the roadmap for a common, open source software infrastructure for scientific computing at exascale.

$\mathrm{He}$ is the coordinator of the Climate-G Project.

He is the author of more than 100 papers in refereed journals on parallel $\&$ grid computing.

MARCO PULIMENO received a Bachelor's Degree in computer engineering and since 2007 his research interest concerns the Augmented and Virtual Reality technologies applied to medicine, games and edutainment. 